\title{
The Effect of Triangular Dimple Orientation on Load-Carrying Capacity
}

\author{
Yi Xie ${ }^{1, a}$, Xiaohan $\mathrm{Pei}^{1}$ and Songbo Wei ${ }^{1}$ \\ ${ }^{1}$ PetroChina Research Institute of Petroleum Exploration \& Development, Beijing 100083, China
}

\begin{abstract}
In this study, the effect of triangular dimple orientation on load-carrying capacity is further investigated. The effect of geometric and operating parameters including the area ratio, the depth, the angular speed and the film thickness on load-carrying capacity is discussed under different orientation angles. The load-carrying mechanisms of the triangular dimples is analyzed.
\end{abstract}

\section{Introduction}

Surface modified technology is known to improve tribological performances of mechanical components, such as enhancing load-carrying capacity, reducing friction and wear etc. Several surfaces with dimples fabricated by surface modified technology have been applied in mechanical seals[1-5], thrust bearing[6-9], and piston rings[10-11], etc. The area density, the depth and feature distribution of the dimple are important geometric parameters which influence the tribological performance. The tribological performance of surfaces with dimples can be improved by optimizing the above three parameters [1-2,7-8].

In recent years, the effect of the dimple geometric shape on the tribological performance has been the focus of researcher interest, because various dimple shapes can be fabricated with the development of the surface processing methods, such as laser surface texturing, reactive ion etching and abrasive jet machining, etc. There have been many research reports [12-14] about the effect of the dimple geometric shape. At the same time, the triangular dimple with its special nature has aroused the interest of researchers. Siripuram et al.[15] carried out to numerically studied to find that the effect of the triangular dimple orientation on the leakage. Yu et al. [16] carried out theoretical studies to investigate the effect of the triangular dimple orientation on the hydrodynamic pressure. Xie et al. [17] have investigated tribological performance of triangular dimples in water lubrication by experimental methods. Guo et al. [18] have numerically studied the effect of various microdimple shapes on the pumping rate and the friction torque of radial lip seals and found that it was an efficient way to increase pumping rate and decrease friction torque of radial lip seals by changing the triangular dimple orientation. Meng et al. [19] also studied the effect of the triangular dimple orientation on the load-carrying capacity and leakage rate of the mechanical seal. However, it is still necessary for further research about the triangular dimple orientation. It is helpful for understanding the best choice of the triangular dimple orientation and its special properties.

In this paper, the effect of triangular dimple orientation on load-carrying capacity is further investigated. The effect of triangular dimple orientation angles on load-carrying capacity under different geometric and operating parameters including the area ratio, the angular speed, the depth and the film thickness is discussed. The load-carrying mechanisms of the triangular dimples is analyzed.

\section{Theoretical model}

\subsection{Geometric model}

To investigate the effect of triangular dimple orientation on load-carrying capacity, a pair of friction components composed of one upper ring and one lower ring are chosen as shown in Figure 1. The upper ring is the rotating ring and rotates along the anticlockwise direction at an angular speed $\omega$, while the lower ring is the stationary ring. There is a fluid film between the rotating ring and the stationary ring, and the thickness of the film in non-dimple area are is expressed by $h_{0}$. The sizes of the rotating and stationary ring are the same. The outer diameter, $r_{o}$, is $25.4 \mathrm{~mm}$ and the inner diameter, $r_{i}$, is $20.75 \mathrm{~mm}$. The surface roughness of the rotating ring is $0.18 \mu \mathrm{m}$ and the surface roughness of the stationary ring is $0.26 \mu \mathrm{m}$. The triangular dimples are radioactively distributed on the stationary ring. The dimple numbers in circumferential and radial direction are 80 and 4, respectively. The dimple bottom is flat, and the dimple depth is expressed by $h_{\mathrm{g}}$.

As is well known[1-2], the area density of dimples is an important parameter which affects the performance of dimples. So in this study the area density for triangular dimple can be defined as

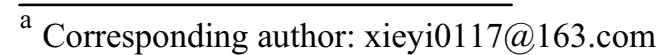




$$
S_{\mathrm{p}}=\frac{A_{\mathrm{p}}}{A}=\frac{n_{\mathrm{r}} n_{\mathrm{c}} a b}{2 \pi\left(r_{\mathrm{o}}^{2}-r_{\mathrm{i}}^{2}\right)}
$$

where $A_{\mathrm{p}}$ is the total dimple area, $A$ is the total surface area, $n_{r}$ is the dimple number in circumferential direction, $n_{c}$ is the dimple number in radial direction, $a$ is the bottom of the triangular dimple, $b$ is the height of the triangular dimple.

The inclination of the triangular dimple is described by an orientation angle, $\alpha$, which is shown in Figure 1. The shape dimension parameter, $\gamma$, can be described by the ratio of the height to the bottom length in the following

$$
\gamma=\frac{b}{a}
$$

The nominal film thickness equation can be expressed in the following:

$$
h(x, y)=\left\{\begin{array}{cc}
h_{0}+h_{\mathrm{g}} & (x, y) \in \text { dimple area } \\
h_{0} & \text { else }
\end{array}\right.
$$

where $x$ and $y$ are the coordinates in the surface with triangular dimple.

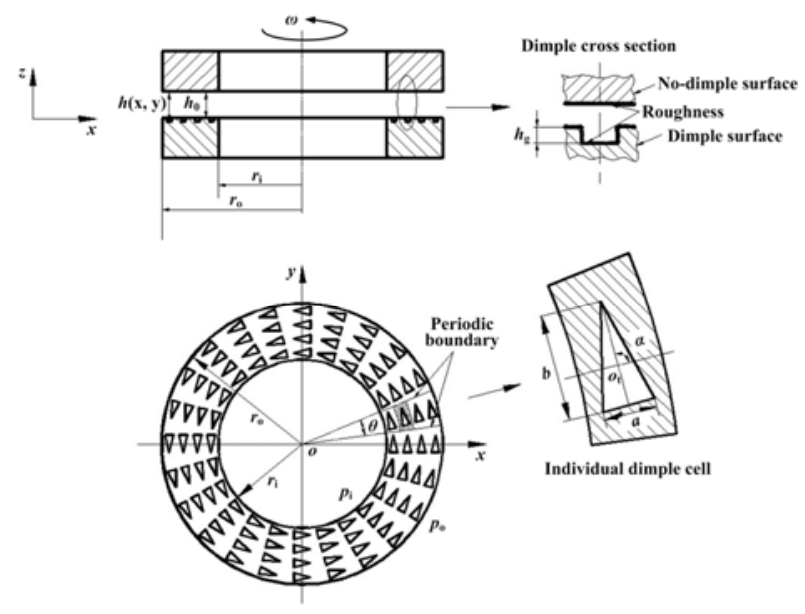

Figure 1. Structure schematic of a pair of friction with triangular dimples.

\subsection{Mathematical model}

In this study, the fluid between the rotating ring and the stationary ring is assumed to be laminar, incompressible Newtonian fluid. The hydrodynamic pressure can be solved by the Reynolds equation. According to the average flow model proposed by Patir and Cheng [2021], the Reynolds equation which satisfies the mass conservation can be written in the Cartesian coordinate in the following:

$$
\begin{aligned}
& \frac{\partial}{\partial x}\left(\phi_{x} \frac{\rho h^{3}}{12 \mu} \frac{\partial p}{\partial x}\right)+\frac{\partial}{\partial y}\left(\phi_{y} \frac{\rho h^{3}}{12 \mu} \frac{\partial p}{\partial y}\right)= \\
& \frac{\partial}{\partial x}\left[\frac{U}{2} \rho\left(h_{T}+\sigma \phi_{s}\right)\right]+\frac{\partial}{\partial y}\left[\frac{V}{2} \rho\left(h_{T}+\sigma \phi_{s}\right)\right]+\frac{\partial\left(\rho h_{T}\right)}{\partial t}
\end{aligned}
$$

where $p$ is the hydrodynamic pressure; $h_{T}$ is the average local film thickness; $U$ and $V$ are respectively the speeds of the rotating ring in the $x$ and $y$ directions; $\phi_{x}$ and $\phi_{y}$ are respectively the pressure flow factors in the $x$ and $y$ directions; $\phi_{s}$ is the shear flow factor. $\rho$ and $\mu$ are the viscosity and density of the fluid film. In the process of numerical simulation, the effect of the squeeze is not taken into account, so the squeeze term, $\partial h_{T} / \partial t$, is neglected.

\subsection{Boundary condition}

The periodic boundary condition and the boundary condition at the inner and outer diameters in computational domain are given by

$$
p_{\theta-}=p_{\theta+}
$$

where $\theta-$ is the initial boundary of triangular dimple column, and $\theta+$ is the final boundary of triangular dimple column.

The fluid pressure at the inner and outer diameters is assumed to be constant, so the boundary condition at the inner and outer diameters can be given by

$$
\left\{\begin{array}{l}
p\left(\sqrt{x^{2}+y^{2}}=r_{\mathrm{o}}\right)=p_{\mathrm{o}} \\
p\left(\sqrt{x^{2}+y^{2}}=r_{\mathrm{i}}\right)=p_{\mathrm{i}}
\end{array}\right.
$$

where $p_{\mathrm{o}}$ is the outside pressure and $p_{\mathrm{i}}$ is the inside pressure.

It is known that when the fluid local pressure falls below the cavitation pressure in operation, the fluid film will rupture and the cavitation occurs. It is very necessary to use an appropriate boundary condition for the cavitation problem. In the present, there are three boundary conditions: the half-Sommerfeld boundary condition, the Reynolds boundary condition and the JFO boundary condition. The JFO boundary condition which satisfies the mass conservative law is considered as an accurate method for handling the cavitation problem [2223]. Therefore, the method in this study is based on the FEM proposed by Kumar and Booker [24]. The basic assumptions for the fluid film are: 1) the fluid film consists of the bi-phase lubricant mixture, including liquid and vapor or gas; 2) the liquid in full film zones is assumed to be incompressible, while the gas/vapor in the cavitation zones is assumed to be compressible with zero bulk modulus; 3) the density and the viscosity of gas/vapor in the cavitation zones are assumed to be zero. According to the basic assumptions, the relationship of the local viscosity and density of the bi-phase mixture can be shown in the following

$$
\mu / \mu_{\text {liq }}=\rho / \rho_{\text {liq }}
$$

where $0 \leq \rho \leq \rho_{\text {liq }}, 0 \leq \mu \leq \mu_{\text {liq }} . \rho$ and $\mu$ are the viscosity and density of the bi-phase mixture. $\rho_{\text {liq }}$ and $\mu_{\text {liq }}$ are the density and the viscosity of the liquid in full film zones.

\subsection{Dimensionless form}

Under the above basic assumptions, a dimensionless form equation is expressed in the following 


$$
\begin{aligned}
& \frac{\partial}{\partial x^{*}}\left(\phi_{x} h^{* 3} \frac{\partial p^{*}}{\partial x^{*}}\right)+\frac{\partial}{\partial y^{*}}\left(\phi_{y} h^{* 3} \frac{\partial p^{*}}{\partial y^{*}}\right)= \\
& \Lambda\left\{\begin{array}{l}
\frac{\partial}{\partial x^{*}}\left[U^{*} \rho^{*}\left(h_{T}^{*}+\phi_{s}\right)\right]+\frac{\partial}{\partial y^{*}}\left[V^{*} \rho^{*}\left(h_{T}^{*}+\phi_{s}\right)\right] \\
+2 h_{T}^{*} \frac{\partial \rho^{*}}{\partial t}
\end{array}\right\}
\end{aligned}
$$

The dimensionless terms in Eq. 8 are defined as follows

$$
\begin{aligned}
& x^{*}=\frac{x}{r_{\mathrm{i}}}, y^{*}=\frac{y}{r_{\mathrm{i}}}, U^{*}=\frac{U}{r_{\mathrm{i}}}, V^{*}=\frac{V}{r_{\mathrm{i}}}, h^{*}=\frac{h}{\sigma}, p^{*}=\frac{p}{p_{\mathrm{i}}}, \\
& \rho^{*}=\frac{\rho}{\rho_{\mathrm{liq}}}, h_{T}^{*}=\frac{h_{T}}{\sigma}=\frac{h^{*}}{2}\left[1+\operatorname{erf}\left(\frac{h^{*}}{\sqrt{2}}\right)\right]+\frac{1}{\sqrt{2 \pi}} e^{-\frac{h^{* 2}}{2}}, \\
& \Lambda=\frac{6 \mu_{\mathrm{liq}} r_{\mathrm{i}}^{2}}{p_{\mathrm{i}} \sigma^{2}}
\end{aligned}
$$

The dimensionless hydrodynamic pressure $p^{*}$ can be obtained by solving Eq. 8 . The validity of the present method has been verified in ref.[25].

The dimensionless load-carrying capacity $F$ can be expressed in the following

$$
F=\iint_{A} p^{*} d A
$$

\section{Results and discussion}

The following operating parameters and geometrical parameters are used in this study: outside pressure $p_{\mathrm{o}}=0.1 \mathrm{MPa}$, inside pressure $p_{\mathrm{i}}=0.1 \mathrm{MPa}$, cavitation pressure $p_{\mathrm{c}}=0.09 \mathrm{MPa}$, film thickness $h_{0}=5 \mu \mathrm{m}$, angular speed $\omega=1000 \mathrm{rpm}$, viscosity of the liquid $\mu_{\mathrm{liq}}=0.001$ Pa.s, dimple depth $h_{\mathrm{g}}=10 \mu \mathrm{m}$, area density $S_{\mathrm{p}}=20 \%$, ratio of the height to the bottom length $\gamma=0.866$. The parameters will be constant unless they are discussed.
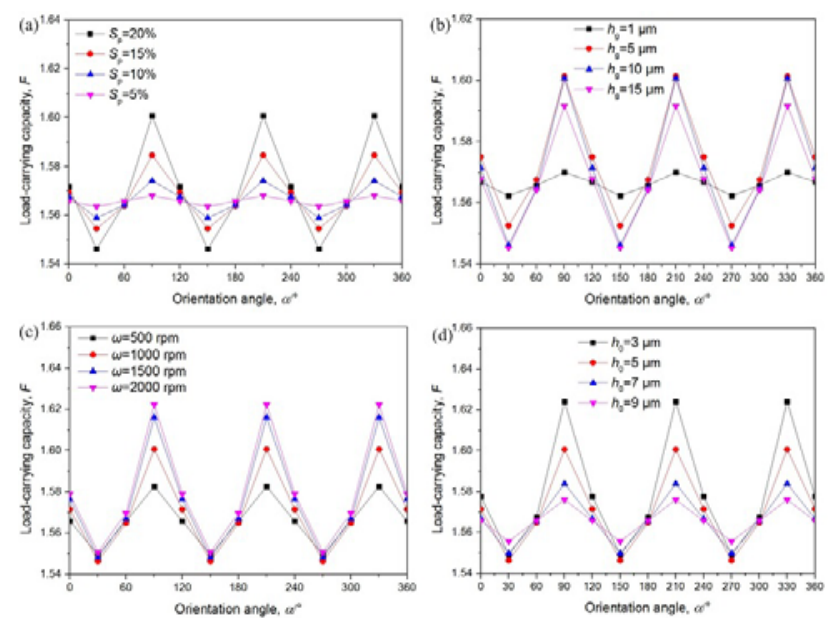

Figure 2. Effect of orientation angles: (a) different area ratios; (b) different dimple depths; (c) different angular speeds; (d) different film thicknesses.

\subsection{Effect of orientation angles under different area ratios}

Figure 2(a) shows the effect of orientation angles under different area ratios. It can be seen that $F$ is dependent on the orientation angles. The $F$ value shows periodic variation with alteration of $\alpha$. So the variation of $F$ value with $\alpha$ can be divided into three periods: $0^{\circ} \sim 120^{\circ}$; $120 \sim 240^{\circ} ; 240 \sim 360^{\circ}$. The maximum values of $F$ appear in $90^{\circ}, 210^{\circ}, 330^{\circ}$, and the minimum values of $F$ appear in $30^{\circ}, 150^{\circ}, 270^{\circ}$ in three periods. So a conclusion can be found that the $F$ could be improved by changing the orientation angles of regular triangular dimple in a period. Furthermore, the value of $F$ is different under the same orientation angle when the area density is different. The larger the value of area density is, the larger the value of $F$ is. In Figure 2(a), when the orientation angles are $90^{\circ}, 210^{\circ}, 330^{\circ}$ and the area density is $20 \%$, the values of $F$ are maximum. So the result shows that the effect of orientation angles on the $F$ is obvious when the area ratio is large.

\subsection{Effect of orientation angles under different depths}

Figure 2(b) shows the effect of orientation angles under different dimple depths. It can be also seen that $F$ is dependent on the orientation angles under different dimple depths. The effect of orientation angles on the $F$ is obvious at a dimple depth and $F$ could be improved by changing the orientation angles of regular triangular dimple. It is indicated that the maximum values of $F$ appear in the dimple depth $h_{\mathrm{g}}=5 \mu \mathrm{m}$ and orientation angle $\alpha=90^{\circ}, 210^{\circ}, 330^{\circ}$.

\subsection{Effect of orientation angles under different angular speeds}

Figure 2(c) shows the effect of orientation angles under different angular speeds. It can be also seen that $F$ is dependent on the orientation angles under different angular speeds. The effect of orientation angles on the $F$ is obvious at an angular speed and $F$ could be improved by changing the orientation angles of regular triangular dimple. But when the angular speed is increased gradually, the incensement rate of $F$ value become small. It is indicated that the effect of the angular speed variation on $F$ is little at an orientation angle when the angular speed is up to $2000 \mathrm{rpm}$.

\subsection{Effect of orientation angles under different film thicknesses}

Figure 2(d) shows the effect of orientation angles under different film thicknesses. It can be also seen that $F$ is dependent on the orientation angles under different film thicknesses. The effect of orientation angles on the $F$ is obvious at a film thickness and $F$ could be improved by changing the orientation angles of regular triangular dimple. Furthermore, the value of $F$ is different under the same orientation angle when the film thickness is different. The smaller the value of film thickness is, the larger the value of $F$ is. 


\section{Conclusions}

The effect of triangular dimple orientation on the loadcarrying capacity is further investigated by numerical simulation, and the conclusions can be drawn.

(1) The load-carrying capacity is dependent on the orientation angles of the triangular dimple. The loadcarrying capacity can be improved by changing the orientation angles of regular triangular dimple.

(2) The value of the load-carrying capacity shows periodic variation with the alteration of the orientation angle for the regular triangular dimple. So the variation can be divided into three periods: $0^{\circ} \sim 120^{\circ} ; 120 \sim 240^{\circ}$; $240 \sim 360^{\circ}$.

(3) The variation of the area ratio, the depth, the angular speed and the film thickness can change the effect of the orientation angles on the load-carrying capacity. Therefore, it is important to consider the variation of the geometric and operating parameters of the triangular dimple when the effect of the orientation angle is investigated.

\section{Acknowledgements}

This project was supported by China Postdoctoral Science Foundation (No. 2015M571063). The authors would also like to thank supports from PetroChina and China National Petroleum Corporation.

\section{References}

1. I. Etsion, L. Burstein, STLE Tribol. Trans. $39677-$ $683(1996)$

2. I. Etsion, Y. Kligerman, STLE Tribol. Trans. 42 511-516 (1999)

3. X.Q. Yu, S. He, R.L. Cai, J. Mater. Process. Technol. 129 463-466 (2002)

4. Y. Wan, D.S. Xiong, J. Mater. Process. Technol. 197 96-100 (2008)

5. S.X. Bai, X.D. Peng, J.Y. Li and S.G. Sheng, STLE Tribol. Lett. 38 187-194 (2010)

6. I. Etsion, G. Halperin, V. Brizmer and Y. Kligerman, STLE Tribol. Lett. 17 295-300 (2004)

7. X.L. Wang, K. Kato, K. Adachi and K. Aizawa, Tribol. Int. 36 189-197 (2003)

8. V. Brizmer, Y. Kligerman, I. Etsion, STLE Tribol. Trans. 46 397-403 (2003)

9. V.G. Marian, M. Kilian, W. Scholz, Proc. Inst. Mech. Eng. Part J: J. Eng. Tribol. 221 771-778 (2007)

10. G. Ryk, I. Etsion, Wear $261792-796$ (2006)

11. I. Etsion, E. Sher, Tribol. Int. 42 542-547 (2009)

12. T. Nanbu, N. Ren, Y. Yasuda, D. Zhu and Q.J. Wang, STLE Tribol. Lett. 29 241-252 (2008)

13. H. Yu, H. Deng, W. Huang and X. Wang, Proc. Inst. Mech. Eng. Part J: J. Eng. Tribol. 225 693-703 (2011)

14. S.X. Bai, X.D. Peng, J.Y. Li and X.K. Meng, Sci. China Technol. Sci. 54 659-662 (2011)

15. R. Siripuram, L.S. Stephens, ASME J. Tribol. 126 527-534 (2004)
16. H.W. Yu, X.L. Wang, F. Zhou, STLE Tribol. Lett. 37 123-130 (2010)

17. Y. Xie, Y.J. Li, Y.M. Wang, S.F. Suo and X.F. Liu, Sci. China phys. mech. 57 273-279 (2014)

18. F. Guo, X.H. Jia, Z. Gao and Y.M. Wang, Sci. China phys. mech. 57 1343-1351 (2014)

19. X.K. Meng, S.X. Bai, X.D. Peng, Tribol. Int. 77 132-141 (2014)

20. N. Patir, H.S. Cheng, ASME J. Lubr. Technol. 100 12-17 (1978)

21. N. Patir, H.S. Cheng, ASME J. Lubr. Technol. 101 220-229 (1979)

22. B. Jakobsson, L. Floberg, Chalmers Tek Hoegsk Handl, 190 1-116 (1957)

23. K.O. Olsson, Trans. Chalmers Univ. Technol. 308 $1-60(1965)$

24. A. Kumar, J.F. Booker, ASME J. Tribol. 113 276$286(1991)$

25. Y. Xie, Y.J. Li, S.F. Suo, X.F. Liu, J.H. Li and Y.M. Wang, Sci. China phys. mech. 56 1909-1919 (2013) 